\title{
The importance of active surveillance in the intensive care unit of Galliera Hospital in Genoa. Analysis of bacterial strains isolated in 2006-2007
}

\section{Maurizio Dallera', David Usiglioº, Paola Fabbri², Nadia Cenderello², Gianluca Ottria', Maria Paola Crisalli', Giovanni Cassola², Maria Luisa Cristina', Paola Sansone², Mauro Nelli', Marco Mori²}

${ }^{1}$ Dipartimento di Scienze della Salute - Laboratorio di Igiene Ospedaliera ed Ambientale, Università degli studi di Genova ²aborartorio di Analisi - E.O. Ospedali Galliera di Genova

KeyWords: Intensive care unit, Surveillance, Antibiotic Resistance

L'importanza della sorveglianza attiva nella Rianimazione dell'E.O. Ospedali Galliera di Genova. Analisi dei ceppi isolati nel biennio 2006-2007

\section{SUMMARY}

Introduction. The risk of infection is higher in intensive care units than in other hospital departments for a number of reasons: the often serious condition of the patients, the invasive diagnostic and therapeutic procedures performed, the indiscriminate use of broad-spectrum antibiotics and the administration of immunosuppressive drugs.

Aims. The present study aimed, on the one hand, to assess the importance of surveillance in the intensive care unit as a means of evaluating healthcare and management procedures and detecting epidemics and "sentinel" microorganisms, and, on the other, to gather up-to-date information on resistance to antibiotics in order to guide proper empirical therapy.

Materials and methods. We conducted a retrospective analysis of the microbiological examinations carried out in the period 2006-2007 in the intensive care unit of $\mathrm{G}$ alliera Hospital in $\mathrm{G}$ enoa. Microbiological surveillance reports and those with antibiograms were picked out.The microorganisms most frequently isolated in the total of samples were listed and then subdivided according to the sample type (respiratory or blood) from which they were isolated. The antibiotic resistance of these microorganisms was subsequently evaluated.

Results. Data analysis revealed that $\mathrm{S}$. aureus was the microorganism most frequently isolated in the total of samples (15.6\%), followed by S. epidermidis (11.6\%) and E. coli (11.1\%).W ith regard to the respiratory samples, S. aureus again proved to be the most frequently isolated strain (18.7\%), while $\mathrm{S}$. epidermidis was isolated from a higher percentage of blood cultures (36.7\%).

Conclusions. The results obtained confirm the utility of infection surveillance procedures in departments at risk, such as intensive care units.

\section{INTRODUZIONE}

Da più di un secolo è noto che l'ospedale può rappresentare un luogo a rischio per l'acquisizione di infezioni da altri pazienti, dal personale, da attrezzature/presidi contaminati o dallo stesso ambiente $(21,22)$

In particolare, le Unità di terapia intensiva (UTI) sono i reparti a più alto rischio di infezioni ospedaliere per il concorrere di molteplici fattori (9) quali le condizioni spesso critiche del paziente, il frequente ricorso a procedure diagnostico-tera- peutiche invasive, la presenza contemporanea in un'area limitata di pazienti altamente suscettibili alle infezioni e di pazienti infetti, l'uso di farmaci immunodepressori e la pressione antibiotica, con la conseguente selezione di microrganismi resistenti difficili da eradicare.

Rispetto ad aree di degenza non intensive, la frequenza di infezioni nosocomiali nelle Unità di terapia intensiva è significativamente più elevata, con una prevalenza pari a $21 \%$ (30) e un'incidenza pari a $34 \%$ (18), anche se i risultati dei diversi

\section{Corresponding author: Maurizio Dallera}

Università degli Studi di Genova, Dipartimento di Scienze della Salute

Via Pastore I - 16132, Genova, Italia.

Tel: +39 0103538857 - Fax: +39 0103538216 - E-mail: maurizio.dallera@unige.it 
studi sono difficilmente confrontabili perché gli indicatori utilizzati sono spesso diversi, e non sempre si è tenuto conto del case mix dei pazienti $(3,18,29,30,33)$

I pazienti ricoverati in UTI, oltre che al rischio di infezioni ospedaliere, sono anche altamente suscettibili alle infezioni sostenute da ceppi resistenti; rispetto a pazienti ricoverati in aree non intensive il rischio di acquisire un patogeno resistente è dal 3\% al 25\% più elevato, a seconda del tipo di microrganismo $(14,15)$.

I pazienti qui ricoverati rappresentano in media solo il 5-10\% del totale, ma sviluppano la metà circa di tutte le infezioni acquisite nel distretto nosocomiale e la relativa mortalità attribuibile alle infezioni è elevata (31).

Dato che non tutte le Infezioni ospedaliere sono prevenibili, i sistemi di sorveglianza delle infezioni ospedaliere (I.O.), a partire dalla fine degli anni 80 , si sono via via concentrati su alcune infezioni associate a specifiche procedure assistenziali.

L'esempio classico di questo approccio alla sorveglianza nelle Unità di Terapia Intensiva è il sistema di sorveglianza statunitense NNIS (National Nosocomial Infection System).

Il pattern epidemiologico delle I.O. nelle unità di terapia intensiva deve essere interpretato come un segnale precoce di allarme poiché alcuni problemi si manifestano inizialmente in tale reparto per poi evidenziarsi in altri (vedi le infezioni correlate ai VRE) $(4,24)$

Contrarre un'infezione durante l'ammissione o il ricovero in rianimazione risulta un evento assai frequente (1) e comporta un aumento della mortalità, durata della degenza e costi (19).

Fin dalla fine degli anni '70 (11) la sorveglianza delle infezioni in terapia intensiva è considerata di fondamentale importanza (8). L'obiettivo di tale pratica si sviluppa nell'identificazione e valutazione di pratiche assistenziali e gestionali al fine di migliorare costantemente l'assistenza (17) permettendo inoltre di evidenziare epidemie, microrganismi "sentinella" nonché di ottenere dati aggiornati sulla antibiotico-resistenza che possono indirizzare correttamente la terapia empirica (28).

Importante sottolineare inoltre che l'attuazione di un programma di sorveglianza porta ad una maggiore attenzione al problema da parte degli operatori sanitari e ricadute positive sui pazienti (16); per tale ragione la sorveglianza può essere considerata una pratica costi/beneficio favorevole (32).

Nei paesi anglosassoni ed in particolare negli Stati Uniti la sorveglianza mira esclusivamente ad evidenziare le infezioni (non le colonizzazio- ni). Il quadro epidemiologico delle infezioni ospedaliere (localizzazioni, agenti responsabili, pattern di resistenza) viene infatti descritto a partire dalle infezioni (13).

Nelle terapie intensive italiane, invece, il termine sorveglianza viene spesso utilizzato in rapporto alle "colture di sorveglianza", cioè alla esecuzione ad intervalli regolari di colture microbiologiche su pazienti asintomatici.

\section{MATERIALI E METODI}

E' stata condotta un'analisi retrospettiva dei risultati relativi agli esami microbiologici eseguiti negli anni 2006-2007 nell'Unità di Terapia Intensiva dell'E.O. Ospedali Galliera di Genova. Tale Unità Operativa è una struttura complessa che gestisce l'attività di degenza in terapia intensiva, l'attività anestesiologica sia di elezione sia di urgenza, l'attività di terapia del dolore e di nutrizione artificiale.

La degenza di rianimazione e terapia intensiva dispone di sette posti letto attrezzati con ventilatori meccanici di ultima generazione, presidi e sistemi di monitoraggio avanzati. Afferiscono alla struttura pazienti critici, che necessitano di supporti artificiali per garantire le funzioni vitali e di uno stretto monitoraggio per consentire tempestive variazioni terapeutiche. La struttura è pluridisciplinare ed è caratterizzata da un'alta specializzazione nel trattamento di gravi e svariate patologie. Per l'Unità Operativa di Rianimazione esiste un protocollo per la diagnosi ed il trattamento delle infezioni in accordo col CIO e la Direzione Sanitaria ed un protocollo relativo alla modalità di attuazione della sorveglianza in tempo reale (26, 27).

Il numero di pazienti ricoverati nell'anno 2006 è stato di 118 mentre nell' anno 2007 sono stati ricoverati 150 pazienti per un totale di 268.

La nostra indagine è iniziata selezionando tutti i referti microbiologici di sorveglianza e con antibiogramma per tutte le tipologie di campioni calcolando il numero di campioni positivi le percentuali di batteri gram positivi e negativi e stilando una lista dei microrganismi maggiormente isolati; successivamente sono state calcolate le percentuali di isolati batteriologici per tipologia di campione: campioni respiratori (bronco aspirati e bronco lavaggi alveolari) e sangue, ed è stata successivamente condotta un'indagine sull'antibiogramma dei microrganismi maggiormente isolati.

L'identificazione e l'antibiogramma dei microrganismi è stato condotto con il metodo automatico Phoenix $^{\circledR}$ (Becton Dickinson). In associazione a tale metodica automatica sono stati abbinati metodi manuali di controllo dei meccanismi di resistenza (metodo della diffusione su disco, E-test 
per la ricerca della ESBL ed MBL ed il controllo sui glicopeptidi, conferme relative a meticillinoresistenza) e sono stati utilizzati alcuni terreni cromogeni per la rilevazione di ceppi MRSA e VRE nelle colture di sorveglianza.

\section{RISULTATI}

I risultati sono stati ottenuti partendo da un numero di campioni pari a 491, in base tale dato è stata calcolata la percentuale di campioni positivi e negativi ed è emersa una positività pari al 60.5\%. Successivamente, tale dato, è stato calcolato discriminando i microrganismi isolati per GRAM negativi (43.7\% ), GRAM positivi (38.1\%), e lieviti (18.3\%), considerando questi ultimi come isolati prevalentemente dalla sorveglianza attiva.

Analizzando la percentuale relativa alla tipologia d'infezione (nosocomiale e comunitaria), come aspettato, le infezioni nosocomiali (68\%) sono risultate maggiori di quelle comunitarie (32\%).

Il passo successivo è stato quello di evidenziare, sulla totalità dei campioni positivi, i microrganismi maggiormente isolati. Dai risultati ottenuti (Tabella 1) è emerso che il microrganismo maggiormente isolato è stato lo $S$. aureus con una percentuale di isolamento pari al $15.6 \%$ (di cui $6.9 \%$ sono risultati MRSA) seguito dallo $S$. epidermidis (11.6\%) e dall'E.coli (11.1\%). Tra gli altri microrganismi isolati si sottolinea P.aeruginosa (7.7\%), Enterobattacter spp (7.7\%), S. maltophilia (4.2\%), H.influenzae (3.2\%).

I dati a disposizione ci hanno consentito di evidenziare la circolazione di S. aureus e calcolare la percentuale di popolazione MRSA presente in rianimazione. Inoltre si è riusciti a stabilire la percentuale di colonizzazioni ed infezioni da parte di tali ceppi escludendo dal calcolo le infezioni acquisite prima dell'ingresso in terapia intensiva. I risultati ottenuti hanno mostrato una percentuale di MRSA pari al 39.4\% di cui il 38.5\% ha sviluppato un'infezione.

Definiti i ceppi maggiormente isolati, sono state calcolate le percentuali dei microrganismi isolati da materiali respiratori e da emocolture. Considerando i campioni respiratori (Tabella 2), provenienti principalmente da colture di sorveglianza, il ceppo maggiormente isolato è risultato essere nuovamente lo S.aureus con una percentuale di isolamento pari al $18.7 \%$ ( di cui $8.0 \%$ MRSA) seguito da C. albicans (13.7\%) e da $S$. epidermidis (10.7\%). Inoltre, tra gli altri isolati, sono state riscontrate percentuali rilevanti per $P$. aeruginosa (8.0\%), E.coli (5.7\%) e S. maltophilia (5.3\%).

La Tabella 3 mostra i ceppi più frequentemente isolati nelle emocolture; in questo caso $S$. epidermidis è stato il microrganismo isolato con per- centuale più elevata (36.7\%) seguito da C. albicans $(16.7 \%)$ e dallo $S$. aureus $(13.4 \%$ di cui la metà MRSA). Importante il dato relativo alle infezioni CVC correlate in quanto è stata evidenziata una percentuale pari al 9.9\% sul totale delle emocolture inviate.

Determinata la circolazione globale dei microrganismi in rianimazione ed evidenziati i ceppi maggiormente isolati nel distretto respiratorio e nel torrente circolatorio sono stati analizzati gli antibiogrammi relativi ad alcuni dei principali batteri di interesse patogeno.

L'analisi dell'antibiogramma relativo allo $S$. aureus (Tabella 4) ha mostrato ceppi MRSA pari al 38.1\% ed una resistenza in evoluzione ai chinolonici (Levofloxacina 23.8\%, Ciprofloxacina $35.7 \%)$. Resistenza relativamente bassa è stata evidenziata per l'Eritromicina (14.3\%); per quanto riguarda gli antibiotici salvavita (Linezolide, Quinupristina/Dalfopristina) e i glicopeptidi non è stata rilevata alcuna resistenza.

Interessante sottolineare il dato relativo all'incidenza di S. aureus per l'anno 2007, che ha mostrato nel $2^{\circ}$ trimestre una percentuale di MRSA pari al 55.5\%; mentre negli altri trimestri l'incidenza è stata sempre inferiore (minimo $33.3 \%$ nel $1^{\circ}$ trimestre massimo $40.0 \%$ nel $3^{\circ}$ e $4^{\circ}$ trimestre)

In Tabella 5 è stato riportato l'antibiogramma relativo a $S$. epidermidis il quale ha evidenziato un'elevata percentuale di resistenza ai chinolonici (Levofloxacina 75.8\%, Ciprofloxacina 81.8\%) ed all'Oxacillina $(72.7 \%)$. Da sottolineare una resistenza pari al 51.5\% relativa al'Eritromicina. Non è stata rilevata alcuna resistenza per Vancomicina (presenza di alcuni ceppi con MIC spostata al $4 \mu \mathrm{g} / \mathrm{ml})$, Teicoplanina, Linezolide e Quinupristina/Dalfopristina.

L'antibiotico-resistenza relativa a $E$. coli è stata descritta in Tabella 6. I risultati ottenuti mostrano una resistenza ai chinolonici variabile dal $20 \%$ per la Levofloxacina fino al $47.1 \%$ per l'acido nalidixico. Per quanto riguarda Piperacillina e Ampicillina la soglia di resistenza si stabilizza rispettivamente al $42.2 \%$ ed al $53.3 \%$. I ceppi produttori di ESBL sono risultati pari al 9.5\% (dato in evoluzione).

In Tabella 7 è stata riportata l'antibiotico-resistenza relativa a $P$. aeruginosa. Il dato interessante riguarda i carbapenemici per i quali la resistenza riscontrata è stata pari al 23.3\% per l'Imipenem. In ultima analisi è stato riportato in Tabella 8 l'antibiogramma relativo a $S$. maltophilia, un microrganismo intrinsecamente resistente ad un pool di antibiotici assai vasto. Per ciò che riguarda gli antibiotici di maggior utilizzo per tale microrganismo, un risultato importante si evince osservando la resistenza al Cotrimossazolo (38.5\% in aumen- 
to) ed ai chinolonici con una percentuale di resistenza alla Levofloxacina pari al $23.1 \%$ ed alla Ciprofloxacina 69.2\%. Va sottolineato che quasi tutta la totalità di ceppi analizzati proveniva da colonizzazioni del tratto respiratorio per i quali si è concordato di fornire eccezionalmente l'antibiogramma vista la frequenza di isolamento.

Tabella 1. Percentuali relative ai ceppi maggiormente isolati sul totale dei campioni

\begin{tabular}{lc}
\hline Ceppo & \%isolati \\
\cline { 2 - 2 } S. aureus & 15.6 \\
\hline S. epidermidis & Di cui MRSA: \\
\hline E. coli & 11.6 \\
\hline C. albicans & 11.1 \\
\hline P.aeruginosa & 10.8 \\
\hline Enterobacter spp & 7.7 \\
\hline Klebsiella spp & 7.7 \\
\hline C.glabrata & 5.0 \\
\hline S. maltophilia & 5.0 \\
\hline H. influenzae & 4.2 \\
\hline E.faecium & 3.2 \\
\hline E. faecalis & 2.1 \\
\hline
\end{tabular}

Tabella 2. Percentuali relative ai ceppi maggiormente isolati in nei campioni respiratori

\begin{tabular}{lc}
\hline Ceppo & \%di isolati \\
\cline { 2 - 2 } S. aureus & 18.7 \\
\hline C. albicans & Di cui MRSA: \\
\hline S. epidermidis & 13.7 \\
\hline P.aeruginosa & 10.7 \\
\hline E.coli & 8.0 \\
\hline S. maltophilia & 5.7 \\
\hline C.glabrata & 5.3 \\
\hline E. cloacae & 5.3 \\
\hline K. pneumoniae & 5.0 \\
\hline H. influenzae & 4.6 \\
\hline
\end{tabular}

Tabella 3. Percentuali relative ai ceppi maggiormente isolati nelle emocolture e percentuale di CVC correlate

\section{Ceppo}

S. epidermidis

C. albicans

S. aureus

E. coli

E. faecalis

E.faecium

A. baumannii

CVC CORRELATE
C. parapsilosis \%di isolati

$$
36.7
$$

16.7

13.4

Di cui MRSA:

6.7

10.0

6.7

6.7

6.7

3.3
Tabella 4. Percentuali di antibiotico-resistenza in S.aureus

\section{Antibiotico}

Percentuale di resistenza (\%)

\begin{tabular}{lc}
\hline Amoxi-clavulanato & 47.6 \\
\hline Ampicillina & 85.7 \\
\hline Cefazolina & 38.1 \\
\hline Cefoxitina & 38.1 \\
\hline quinupristina/dalfo pristina & 0.0 \\
\hline Ciprofloxacina & 35.7 \\
\hline Clindamicina & 11.9 \\
\hline Cloramfenicolo & 19.0 \\
\hline Eritromicina & 14.3 \\
\hline Gentamicina & 21.4 \\
\hline Levofloxacina & 23.8 \\
\hline Linezolide & 0.0 \\
\hline Oxacillina & 38.1 \\
\hline Penicillina G & 85.7 \\
\hline Rifampicina & 2.4 \\
\hline Teicoplanina & 0.0 \\
\hline Trimethoprim/sulfametossazolo & 0.0 \\
\hline Vancomicina & 0.0 \\
\hline
\end{tabular}

Tabella 5. Percentuali di antibiotico-resistenza in S. epidermidis

\begin{tabular}{lc}
\hline Antibiotico & $\begin{array}{c}\text { Percentuale di } \\
\text { resistenza (\%) }\end{array}$ \\
\hline Amoxi-clavulanato & 75.8 \\
\hline Ampicillina & 96.8 \\
\hline Cefazolina & 72.7 \\
\hline Cefoxitina & 72.7 \\
\hline quinupristina/dalfo pristina & 0.0 \\
\hline Ciprofloxacina & 81.8 \\
\hline Clindamicina & 40.7 \\
\hline Cloramfenicolo & 15.2 \\
\hline Eritromicina & 51.5 \\
\hline Gentamicina & 33.3 \\
\hline Levofloxacina & 75.8 \\
\hline Linezolide & 0.0 \\
\hline Oxacillina & 72.7 \\
\hline Penicillina G & 90.9 \\
\hline Rifampicina & 27.3 \\
\hline Teicoplanina & 0.0 \\
\hline Trimethoprim/sulfametossazolo & 40.6 \\
\hline Vancomicina & 0.0 \\
\hline
\end{tabular}

\section{CONCLUSIONI}

I risultati ottenuti in questo lavoro confermano l'utilità delle procedure di Sorveglianza delle Infezioni in reparti di degenza quali la Rianimazione (Unità di terapia intensiva).

In letteratura si dibatte molto sull'utilità delle colture microbiologiche di sorveglianza $(2,5,6,7$, 20, 25), nella nostra esperienza ha effettivamente evidenziato un discreto valore prognostico soprattutto nei casi di evoluzione da colonizzazione ad infezione relativi a ceppi MRSA (38.1\%). 
Tabella 6. Percentuali di antibiotico-resistenza in E. coli

\begin{tabular}{lc}
\hline Antibiotico & $\begin{array}{c}\text { Percentuale di } \\
\text { resistenza (\%) }\end{array}$ \\
\hline Acido nalidixico & 47.1 \\
\hline Amikacina & 0.0 \\
\hline Amoxi-clavulanato & 20.0 \\
\hline Ampicillina & 53.3 \\
\hline Aztreonam & 11.1 \\
\hline Cefazolina & 11.1 \\
\hline Cefepime & 10.7 \\
\hline Cefotaxima & 10.7 \\
\hline Ceftazidima & 11.1 \\
\hline Ceftriaxone & 11.8 \\
\hline Cefuroxime sodico & 11.8 \\
\hline Ciprofloxacina & 22.2 \\
\hline Fosfomicina c/G 6PD & 0.0 \\
\hline Cloramfenicolo & 21.4 \\
\hline Gentamicina & 8.9 \\
\hline Imipenem & 0.0 \\
\hline Levofloxacina & 20.0 \\
\hline N itrofurantoina & 0.0 \\
\hline N orfloxacina & 35.3 \\
\hline Piperacillina & 42.2 \\
\hline Pipera/tazobactam & 6.7 \\
\hline Tetraciclina & 50.0 \\
\hline Trimethoprim/sulfametossazolo & 24.4 \\
\hline ESBL & 9.5 \\
\hline
\end{tabular}

Tabella 7. Percentuali di antibiotico-resistenza riscontrati in P.aeruginosa

\begin{tabular}{lc}
\hline Antibiotico & $\begin{array}{c}\text { Percentuale di } \\
\text { resistenza (\%) }\end{array}$ \\
\hline Aztreonam & 20.0 \\
\hline C efepime & 13.3 \\
\hline Ceftazidima & 13.3 \\
\hline Ciprofloxacina & 13.3 \\
\hline Gentamicina & 13.3 \\
\hline Imipenem & 23.3 \\
\hline Levofloxacina & 20.0 \\
\hline Pipera/tazobactam & 3.3 \\
\hline
\end{tabular}

Tabella 8. Percentuali di antibiotico-resistenza riscontrati in S.maltophilia

\begin{tabular}{lc}
\hline Antibiotico & $\begin{array}{c}\text { Percentuale di } \\
\text { resistenza (\%) }\end{array}$ \\
\hline Ciprofloxacina & 69.2 \\
\hline Levofloxacina & 23.1 \\
\hline Trimetho prim/sulfametossazolo & 38.5 \\
\hline
\end{tabular}

Abbiamo invece constatato una notevole ricaduta sull'attenzione degli operatori verso le problematiche relative alle infezioni ospedaliere e alla circolazione di ceppi potenzialmente patogeni e di facile trasmissione (MRSA. Bacilli gram negativi non fermentanti e altri), di inestimabile valore formativo e preventivo.

Confrontando la letteratura disponibile è stato evi- denziato che dal punto di vista epidemiologico i nostri risultati sono sovrapponibili seppur con qualche differenza relativa ai diversi bacini di utenza.

Per quanto riguarda i risultati ottenuti sottolineiamo la problematica relativa alle infezioni CVC correlate con percentuale pari al 9.9\%, che ha stimolato interventi specifici di prevenzione e controllo della gestione del CVC stesso.

Anche l'ambiente gioca un ruolo assai importante in unità di terapia intensiva, soprattutto in relazione all'isolamento di potenziali patogeni tipicamente ambientali (bacilli gram negativi non fermentanti) quali lo $S$. maltophilia. Studi recenti, hanno sottolineato il possibile impatto, in termini di morbilità e mortalità, di un infezione da $S$. maltophilia, in particolare nei pazienti dei reparti di cure intense con elevato rischio infettivo, che presentano patologie gravi o un'immunosoppressione. La confusione fra colonizzazione e infezione è stata probabilmente favorita da alcuni studi nei quali l'infezione da S. maltophilia non era associata ad un'importante morbilità o mortalità, oppure dal fatto che un'infezione da $S$. maltophilia non era considerata possibile se non in sinergia con altri batteri. La resistenza di S. maltophilia a numerosi antibiotici è un ulteriore fattore di rischio. Attualmente la situazione si fa ancora più inquietante, a causa dell `apparire di ceppi multiresistenti, anche al trimethoprim-sulfametossazolo, l'antibiotico che viene abitualmente prescritto nel trattamento delle infezioni da S. maltophilia $(10,12,23)$; nel nostro caso la resistenza (nei ceppi prevalentemente da colonizzazione) arriva fino al 38.5\%.

L'aumento dell'incidenza di isolamenti di S. maltophilia riscontrati nel biennio in esame ci ha indotto alla revisione delle procedure infermieristiche (nel reparto di rianimazione) e ci ha portato a sviluppare, con la Direzione Sanitaria, un protocollo che preveda idonei controlli microbiologici ambientali e la tipizzazione molecolare dei ceppi isolati nell'eventualità si verificassero più casi di infezione documentabile.

Osservando i risultati relativi alla circolazione di MRSA si può affermare che tale circolazione appare sotto controllo sebbene nel tempo siano stati registrati picchi che hanno portato a livelli di attenzione sempre alti e soprattutto alla necessità di attivare procedure di prevenzione e controllo al fine di abbattere o quantomeno contenere la circolazione di tali ceppi (39.4\% di MRSA nel biennio 2006-2007 con un 38.5\% di infezioni associate) Il problema legato alla resistenza ai chinolonici riguarda soprattutto i ceppi di $S$. epidermidis i quali mostrano resistenze assai preoccupanti (75.8\% di resistenza alla Levofloxacina e 81.8\% per la Ciprofloxacina) che dovrebbero sensibiliz- 
zare gli operatori sanitari ad un corretto utilizzo di questi antibiotici. Un problema simile ma non ancora a livelli così preoccupanti è stato rilevato dal profilo dell'antibiotico-resistenza ai chinolonici in S. aureus con una resistenza del $23.8 \%$ e $35.7 \%$ rispettivamente per Levofloxacina e Ciprofloxacina. Anche alcuni gram negativi importanti quali l'E.coli hanno mostrato resistenza ai chinolonici ben sviluppata da un minimo del $20 \%$ per la Levofloxacina fino ad un massimo del 47.1\% per l'Acido Nalidixico.

In merito alla circolazione di ceppi produttori di ESBL sono stati rilevati discreti risultati: $9.5 \%$ in E. coli (dato da non sottovalutare). Osservando ora la resistenza ai carbapenemici, particolare attenzione è stata posta verso $P$. aeruginosa il quale non ha però mostrato dati preoccupanti (23.3\% di resistenza per l'Imipenem) anche se da controllare attivamente e costantemente.

Infine per quanto riguarda i cosiddetti farmaci salvavita (Glicopeptidi, Linezolid, Quinupristina/Dalfopristina.) non sono state rilevate resistenze da parte di alcun microrganismo. Anche le MIC per i glicopeptidi non risultano preoccupanti ad esclusione di alcuni S. epidermidis con MIC per Vancomicina spostata a $4 \mu \mathrm{g} / \mathrm{ml}$ (ceppi GISE).

Per concludere, da questo lavoro retrospettivo si può dedurre che una migliore conoscenza dello stato di colonizzazione dei pazienti prima dell'infezione, sia utile nell'instaurare una più efficace terapia, rispetto ad una terapia empirica basata su antibiotici ad ampio spettro, in attesa del referto microbiologico, con positive ripercussioni sul paziente, un abbassamento della pressione selettiva per fenotipi di resistenza e contenimento della spesa farmaceutica.

Per il futuro si auspica il proseguimento degli incontri settimanali del Gruppo Operativo Comitato Infezioni Ospedaliere (GOCIO) con personale medico ed infermieristico della rianimazione quale efficace strumento sia di formazione reciproca sia di prevenzione e controllo delle infezioni nel paziente critico.

\section{BIBLIOGRAFIA}

1. Alberti C, Brun-Buisson C, Burchardi $\mathrm{H}$, et al. Epidemiology of sepsis and infection in ICU patients from an International multicentre color study. Int Care Med 2002; 28:108-22

2. Alvarez-Lerma F, Alvarez B, Luque P, et al. Empiric broad - spectrum antibiotic therapy of nosocomial pneumonia in an intensive care unit: a prospective observational study. Crit. Care 2006; 10(3) R78

3. Apolone G., Melotti R.M., Iapichini G., et al. Cost containment: Europe. Italy. New Horiz 1994; 2 (3): 350-356

4. Aumeran C, Paillard C, Robin F, et al. Pseudomonas aeruginosa and Pseudomonas putida outbreak asso- ciated with contaminated water outlets in an oncohaematology paediatric unit. J Hosp Infect 2007 Jan; 65(1):47-53

5. Blot S, Depuydt P, Vogelaers D, et al. Colonization status and appropriate antibiotic therapy for nosocomial bacteriemia in an intensive care unit. Infect. Control Hosp. Epidemiol 2006 jan; 27(1):104

6. Bollettino Epidemiologico Nazionale Notiziario ISS 2006; 19(6)

7. Bryce EA, SmithJA. Focused microbiological surveillance and gram -negative beta-lactamase-mediated resistance in an intensive care unit. Infect control Hosp Epidemiol 1995 jun; 16(6):331-4

8. Candiani A. Infection survillance in ICU. Minerva Anestesiol 2001;67:39-48

9. Curti C, Ippolito G, Maffei C, et al. Strategie di prevenzione delle infezioni in terapia intensiva. Infezioni in terapia intensiva. Effetti srl ed., Milano 1999, pp. 5-18

10. Denton M, et al. Microbiological and clinical aspects of infection associated with Stenotrophomonas maltophilia. Clin Microbiol Rew 1998; Jan, (1): 57-80

11. Eggimann P, Pitted D.Infection control in the ICU. Chest 2001; 120:2059-93

12. Elting LS, et al. A case-control study of predisposing factors. Inf Control Hosp Epidemiol 1990; 11(3): 134138

13. Emori TG, Culver DH, Horan TC, et al. National Nosocomial infections surveillance system (NNIS): description of surveillance methods. Am J Infect Control 1991 Feb; 19(1): 19-35

14. Fridkin SK, Gaynes RP. Antimicrobial resistance in intensive care units. Clin Chest Med 1999; 20 (2): 303-316

15. Fridkin SK, Steward CD, Edwards JR, et al, and the Project Intensive Care Antimicrobial Resistance Epidemiology (ICARE) Hospitals. Surveillance of antimicrobial use and antimicrobial resistance in United States hospitals: Project ICARE Phase 2. Clin Infect Dis 1999; 29: 245-252

16. Gaynes RP, Solomon S. Improving hospital-acquired infection rates: the CDC experience. Jt Comm J Qual Improv 1996; 22:457-67

17. Haley RW, Culver DH, White JM, Morgan WM, Emori TG, MunnVP et al. The efficay of infection suveillance and control programs in preventing nosocomial infections in US hospital. Am J Epidemiol 1985; 121:182-205

18. Ippolito G., Albertoni F., Dionigi R.V., Giunta F., Melotti R.M., Orefice E. et al. Studio nazionale di incidenza delle infezioni nosocomiali in unità di terapia intensiva. Medico e Paziente 1990; 15: 66-73

19. Jarvis WR. Selected aspects of the socioeconomic impact of nosocomial infections: morbidity, mortality, cost, and prevention. Infect Control Hosp Epidemiol 1996; 17: 552-7

20. Lagmuir AD, Farr W. Founder of modern concepts of surveillance. Int J Epidemiol 1976; 5:13-18

21. Moro M.L., Stazi M.A., Marasca G., Greco D., Zampieri A. National Prevalence Survey of hospital acquired infections in Italy. J Hosp Infect 1983; 8: 7285

22. Moro M.L. Infezioni Ospedaliere. Prevenzione e controllo. 1993 Centro Scientifico Torinese

23. Muder RR. et al. Bacteremia due to Stenotrophomonas ( Xanthomonas) maltophilia: a prospective study of 91 episodes. Clin Inf Dis 1996; 22: 508-512

24. Nseir S, Di Pompeo C, Brisson H, Dewavrin F, Tissier S, Diarra M, Boulo M. Intensive care unit-acquired Stenotrophomonas maltophilia: incidence, risk factors, and outcome. Crit Care 2006;10(5):R143 
25. Orsi GB, Rappono M, Franchi C at al. Surveillance and infection control in an intensive care unit. Infect. Control Hosp. Epidemiol 2005 Mar; 26(3):321-5

26. Protocollo per la diagnosi ed il trattamento delle infezioni in rianimazione e terapia intensiva. E.O. Ospedali Galliera - Genova Struttura Complessa di Anestesia e Rianimazione 2006. Via Mura delle Cappuccine, Genova

27. Protocollo sorveglianza microbiologica in tempo reale. E.O. Ospedali Galliera - Genova Struttura Complessa di Anestesia e Rianimazione 2006. Via Mura delle Cappuccine, Genova

28. Rello J, Sa-Borges M, Correa H, Leal SR, Baraibar J. Variation in etiology of ventilator-associated pneumonia across four treatment sites: implication for antimicrobial prescribing practices. Am J Respir crit Care Med 1999; 160:608-13

29. Richards M.J., Edwards J.R., Culver D.H., Gaynes R.P. Nosocomial infections in medical intensive care units in the United States. National Nosocomial Infections Surveillance System. Crit Care Med 1999; 27: 887-892

30. Vincent J.L., Bihari D.J., Suter P.M., Bruining H.A., White J., Nicolas-Chanoin M.H.,Wolff M., Spencer R.C., Hemmer M. The prevalence of nosocomial infection in intensivecare units in Europe. Results of the European Prevalence of Infection in Intensive Care (EPIC) Study. EPIC International Advisory Committee. JAMA 1995; 274: 639-644

31. Weiss R.A., MC Micheal A.J. Social and environmental risk factors in the emergence of infection diseases. Nat. Med 2004; 10(12): 570

32. Wenzel RP. The economics of nosocomial infections. J Hosp Infect 1995; 31:79-87

33. Wenzel R.P., Thompson R.L., Landry S.M., Russell B.S., Miller P.J., Ponce de Leon S., Miller G.B. Jr. Hospital-acquired infections in intensive care unit patients: An overview with emphasis on epidemics. Infect Control 1983; 4: 371-375 